\title{
Geochemical Characterization of Shallow Lagoon Sediments
}

\author{
Omoregbe, O.A*, Oloyede, D.A**, Ekuerugbe, L.O**, and Nkim, A.G* \\ ${ }^{*}$ Resemco Limited, Port Harcourt \\ ** Geology Department, University of Benin, \\ DOI: 10.29322/IJSRP.10.05.2020.p10148 \\ http://dx.doi.org/10.29322/IJSRP.10.05.2020.p10148
}

\begin{abstract}
Geochemical characterization of the Epe lagoon sediments was carried out in order to determine their depositional environment, provenance and weathering history. A total of Nineteen (19) samples were obtained from the upper 2-3m of the lagoon base and analyzed for Elemental composition using the
\end{abstract} Atomic Absorption Spectrophotometry (AAS) method. Laboratory analysis revealed values of the major oxides as follows: $\mathrm{SiO}_{2}(52.99 \%-98.87 \%), \mathrm{Al}_{2} \mathrm{O}_{3}(0.71 \%-35.46 \%), \mathrm{Fe}_{2} \mathrm{O}_{3}$ (0.04-2.96\%), $\mathrm{MgO}(0.01 \%-0.11 \%), \mathrm{CaO}(0.01-0.42), \mathrm{Na}_{2} \mathrm{O}$ (0.01\%-0.08\%), $\mathrm{K}_{2} \mathrm{O}(0.03 \%-0.91 \%), \mathrm{TiO}_{2}(0.01 \%-4.56 \%), \mathrm{P}_{2} \mathrm{O}_{5}$ (0.01\%-0.33), $\mathrm{MnO}(0.01 \%-0.45 \%)$. Very low average values of $\mathrm{Al}_{2} \mathrm{O}_{3} / \mathrm{SiO}_{2}$ (0.08932) and relatively high average value of $\mathrm{SiO}_{2} / \mathrm{Al}_{2} \mathrm{O}_{3}$ (64.33) indicated quartz enrichment which was corroborated by the high $\mathrm{SiO}_{2}$ values recorded. $\mathrm{TiO}_{2} / \mathrm{Al}_{2} \mathrm{O}_{3}$ values (0.004-0.8604) indicated that the sediments are chemically matured. While geochemical analysis using specialized ternary plots revealed that the sediments were fine to medium grained clayey sands deposited in both continental and transitional environments with intensive weathering at the source area.

Index Terms- Geochemical, Lagoon, Provenance, Sediments,

\section{INTRODUCTION}

Geochemical studies have been used extensively in determining concentrations of heavy metals and other harmful elements within sediments. The Lagos lagoon which consists of three main segments (Lagos harbor, the Metropolitan and Epe division) has attracted a lot of geochemical studies arguably because of its location within the commercial city of Lagos which harbours over $75 \%$ of the Industrial outfits of Nigeria (Olatunji and
Abimbola, 2010). However, since geologic processes affect the chemical composition of sediments, geochemical studies have also been widely used in characterizing sediments. Modern approaches in monitoring sediment provenance especially through discriminatory diagrams based on major and trace elements relationship has proved effective (Bauluz et.al, 2000). The geochemical behaviour of elements within sediments indicates substantial changes in the physical, chemical and biological conditions of the environment (Avramidis et. al, 2010). Although various studies have been carried out by several workers in the Lagos Lagoon (Badejo et.al, 2014; Olatunji and Abimbola, 2010; Balogun et.al, 2011; Aderinola et.al, 2009; Ayola and Kuton 2009; Don-pedro et.al, 2004; Okoye et.al, 1991; Ogunsua et.al, 1993; Osibanjo and Bamgbose, 1990; Oyewo et.al, 1982), not much emphasis have been made on the Epe division segment. Also much of the previous studies have focused on using geochemical data to ascertain the impact of both Industrial and anthropogenic waste on the sediments as well as aquatic life.

This current study would focus on using geochemical data in characterizing sediments of the Epe division segment of the Lagos Lagoon in order to determine their depositional environment, provenance, weathering history and also update on existing available geochemical information.

\section{The study Area}

The Epe division segment of the Lagos lagoon (also called the Epe lagoon) lies within Latitude $6^{\circ} 29^{\prime} \mathrm{N}$ to $6^{\circ} 38^{\prime} \mathrm{N}$ and Longitude $3^{\circ} 30^{\prime} \mathrm{E}$ to $4^{\circ} 05^{\prime} \mathrm{E}$. It is located in Lagos, one of Africa's densely populated cities. The lagoon is fed by river Oshun and opens into 
the gulf of guinea through the Lagos harbour. It also has a surface area of about $225 \mathrm{~km}^{2}$ with maximum depth of about $6 \mathrm{~m}$. However, a large area of the lagoon is relatively shallow with minimum depth of about $1 \mathrm{~m}$. Geologically, the Epe lagoon lies within the eastern axis of the Dahomey sedimentary Basin. The regional geologic setting, basin evolution, tectonic elements, stratigraphy and structural styles of the Eastern Dahomey Basin has been dealt with by various authors (Storey, 1995; Mpanda, 1997; Omatsola and Adegoke, 1981; Ojeda, 1982; Adediran and Adegoke, 1987; Slansky, 1962; Billman, 1992; Adegoke, 1969; Ogbe, 1972; Agagu 1985).

\section{MATERIALS AND METHODS}

\section{Sample collection}

A total of nineteen (19) samples were obtained from the upper 2$3 \mathrm{~m}$ of the lagoon base (i.e., the sediment-water interface) with sample points appropriately located using the Global Positioning System (Fig.1) (Table 1).

\section{Sample preparation and sieving}

After collection, the samples were drained of water and air-dried for one week. The dried samples were then disaggregated and the unwanted materials subsequently removed. In order to determine the granulometric characteristics of the samples, $100 \mathrm{~g}$ of airdried soil sample was soaked in 1 litre solution of distilled water with $2 \mathrm{~g}$ of calgon and the whole content was shaken regularly and agitated to ensure effective disaggregation. The soaked sample was then left for 24 hours and thereafter washed through sieve $2.36 \mathrm{~mm}$ opening a little at a time. The washing continued until the water passing through the sieve was clear. The soil retained on the sieve was oven dried at a temperature of $100^{\circ} \mathrm{c}$ to $110^{\circ} \mathrm{c}$ for 24 hours and then subjected to mechanical analysis.

The mechanical analysis of the samples involved the determination of particle size distribution for coarse grained soil i.e. grains coarser than $0.063 \mathrm{~mm}$ using a set of sieves. The sieves were carefully cleaned and their corresponding weight recorded. The collecting pan was placed at the bottom and the sieves arranged in order of increasing mesh size with the finest at the bottom and the coarsest at the top. The oven dried soil was then poured into the sieve from the top, covered with a lid and the whole stack taken to the sieve shaker which shook for 10 minutes

This publication is licensed under Creative Commons Attribution CC BY.

http://dx.doi.org/10.29322/IJSRP.10.05.2020.p10148 thereafter the stack of sieves was removed from the sieve shaker and the amount retained on each sieve was weighed and the weight recorded. The percentage on each sieve was then computed by dividing the weight retained on each sieve with the original soil weight.

\section{Laboratory Analysis}

Inorganic geochemical analysis which involved digestion and determination of major and trace element abundances using the Atomic Absorption Spectrophotometry (AAS) method was carried out on the sediments.

\section{RESULTS AND DISCUSSIONS}

\section{Lithologic Description}

The sediments of the Epe lagoon were mainly fine to medium grained clayey-sands ranging from light to dark grey in colour with some organic debris as well as some fossil shells. Although, some very fine clay materials with preponderant organic debris were also found (Table 2). Majority of the sediments plotted within the clayey-sand region very close to the sand axis indicating a dominance of sandy materials within the sediments (Fig. 2), as confirmed by the high $\mathrm{SiO}_{2}$ values (Table 3). The presence of organic debris within the sediments (Table 2) can be attributed to decay of both dead plant and animal matter which may have been transported from adjoining rivers, deposited within the lagoon and accumulated within the sediments.

\section{Geochemical Analysis}

Whole rock geochemistry of the study area is discussed mainly under major elements through the use of various ternary and bivariate plots which help in classification as well as deducing the depositional environment and weathering history of the sediments of the Epe lagoon.

\section{Major Element Geochemistry}

The abundance of major elements in the sediments of the Epe lagoon as well as their tabulated range of values and calculated mean values as obtained from the major element geochemical analysis are listed in Table's 3, 4, 5 and 6. The dominance of $\mathrm{SiO}_{2}$ over all other oxides is a confirmation of the greater proportions of sandy materials within the sediments. The sediments have average $\mathrm{SiO}_{2}$ values higher than the average 


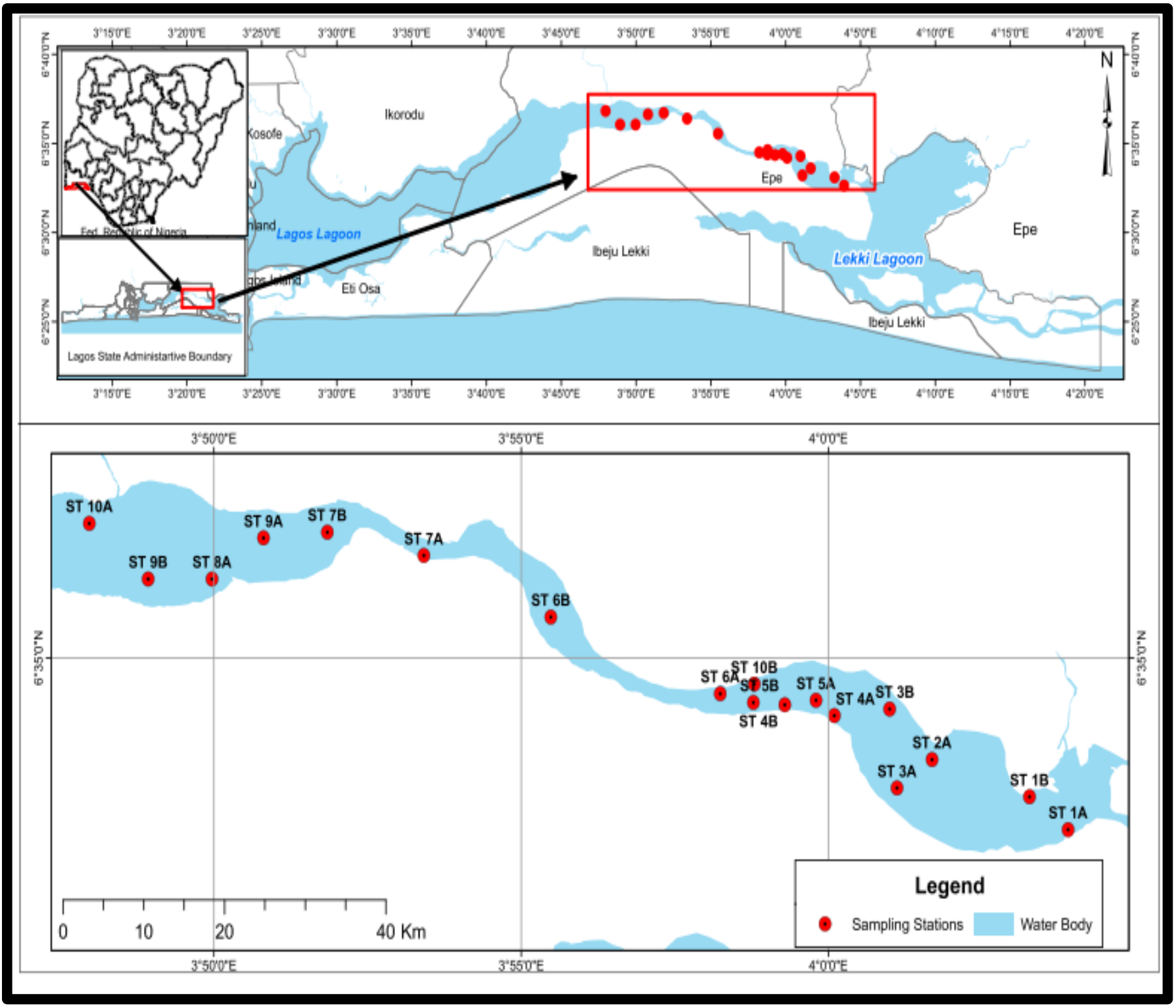

Fig. 1: Map of study area showing sample locations

TABLE 1: GPS Coordinates of sample points and locations within the study area

\begin{tabular}{|c|c|c|c|c|}
\hline S/N & SAMPLE NUMBER & LOCATION & LATITUDE & LONGITUDE \\
\hline 1 & Epe/st/1b & Epe lagoon & $6^{\circ} 33^{\prime} 27.74^{\circ} \mathrm{N}$ & $4^{\circ} 03^{\prime} 21.51^{\circ} \mathrm{E}$ \\
\hline 2 & Epe/st/10a & Epe lagoon & $6^{\circ} 37^{\prime} 00.10^{\circ} \mathrm{N}$ & $3^{\circ} 46^{\prime} 32.02^{\circ} \mathrm{E}$ \\
\hline 3 & Epe/st/7a & Epe lagoon & $6^{\circ} 36^{\prime} 38.63^{\circ} \mathrm{N}$ & $3^{\circ} 51^{\prime} 50.86^{\circ} \mathrm{E}$ \\
\hline 4 & Epe/st/4b & Epe lagoon & $6^{\circ} 34^{\prime} 18.29^{\circ} \mathrm{N}$ & $3^{\circ} 59^{\prime} 45.66^{\circ} \mathrm{E}$ \\
\hline 5 & Epe/st/1a & Epe lagoon & $6^{\circ} 32^{\prime} 39.88^{\circ} \mathrm{N}$ & $4^{\circ} 03^{\prime} 54.85^{\circ} \mathrm{E}$ \\
\hline 6 & Epe/st/4a & Epe lagoon & $6^{\circ} 34^{\prime} 49.34^{\circ} \mathrm{N}$ & $3^{\circ} 59^{\prime} 23.79^{\circ} \mathrm{E}$ \\
\hline 7 & Epe/st/8b & Epe lagoon & $6^{\circ} 36^{\prime} 02.41^{\circ} \mathrm{N}$ & $3^{\circ} 49^{\prime} 17.74^{\circ} \mathrm{E}$ \\
\hline 8 & Epe/st/3a & Epe lagoon & $6^{\circ} 33^{\prime} 15.64^{\circ} \mathrm{N}$ & $4^{\circ} 01^{\prime} 07.48^{\circ} \mathrm{E}$ \\
\hline 9 & Epe/st/5b & Epe lagoon & $6^{0} 34^{\prime} 32.26^{\circ} \mathrm{N}$ & $3^{\mathrm{O}} 58^{\prime} 17.06^{\circ} \mathrm{E}$ \\
\hline
\end{tabular}




\begin{tabular}{|c|c|c|c|c|}
\hline 10 & Epe/st/7b & Epe lagoon & $6^{\mathrm{O}} 36^{\prime} 04.42^{\circ} \mathrm{N}$ & $3^{\mathrm{O}} 49^{\prime} 57.97^{\circ} \mathrm{E}$ \\
\hline 11 & Epe/st/3b & Epe lagoon & $6^{\mathrm{O}} 34^{\prime} 11.56^{\mathrm{O}} \mathrm{N}$ & $4^{\mathrm{O}} 00^{\prime} 06.70^{\circ} \mathrm{E}$ \\
\hline 12 & Epe/st/9a & Epe lagoon & $6^{\mathrm{O}} 36^{\prime} 48.28^{\mathrm{O}} \mathrm{N}$ & $3^{\mathrm{O}} 47^{\prime} 58.18^{\mathrm{O}} \mathrm{E}$ \\
\hline 13 & Epe/st/9b & Epe lagoon & $6^{\mathrm{O}} 36^{\prime} 40.01^{\mathrm{O}} \mathrm{N}$ & $3^{\mathrm{O}} 48^{\prime} 38.14^{\mathrm{O}} \mathrm{E}$ \\
\hline 14 & Epe/st/5a & Epe lagoon & $6^{\mathrm{O}} 34^{\prime} 18.59^{\mathrm{O}} \mathrm{N}$ & $3^{\mathrm{O}} 58^{\prime} 47.62^{\mathrm{O}} \mathrm{E}$ \\
\hline 15 & Epe/st/2a & Epe lagoon & $6^{\mathrm{O}} 33^{\prime} 39.23^{\mathrm{O}} \mathrm{N}$ & $4^{\mathrm{O}} 01^{\prime} 43.03^{\mathrm{O}} \mathrm{E}$ \\
\hline 16 & Epe/st/8a & Epe lagoon & $6^{\mathrm{O}} 36^{\prime} 36.01^{\mathrm{O}} \mathrm{N}$ & $3^{\mathrm{O}} 50^{\prime} 48.30^{\circ} \mathrm{E}$ \\
\hline 17 & Epe/st/6a & Epe lagoon & $6^{\mathrm{O}} 35^{\prime} 36.11^{\mathrm{O}} \mathrm{N}$ & $3^{\mathrm{O}} 55^{\prime} 27.72^{\mathrm{O}} \mathrm{E}$ \\
\hline 18 & Epe/st/6b & Epe lagoon & $6^{\mathrm{O}} 36^{\prime} 19.03^{\circ} \mathrm{N}$ & $3^{\mathrm{O}} 53^{\prime} 32.66^{\circ} \mathrm{E}$ \\
\hline 19 & Epe/st/10b & Epe lagoon & $6^{\mathrm{O}} 34^{\prime} 33.45^{\circ} \mathrm{N}$ & $3^{\mathrm{O}} 58^{\prime} 42.63^{\mathrm{O}} \mathrm{E}$ \\
\hline
\end{tabular}

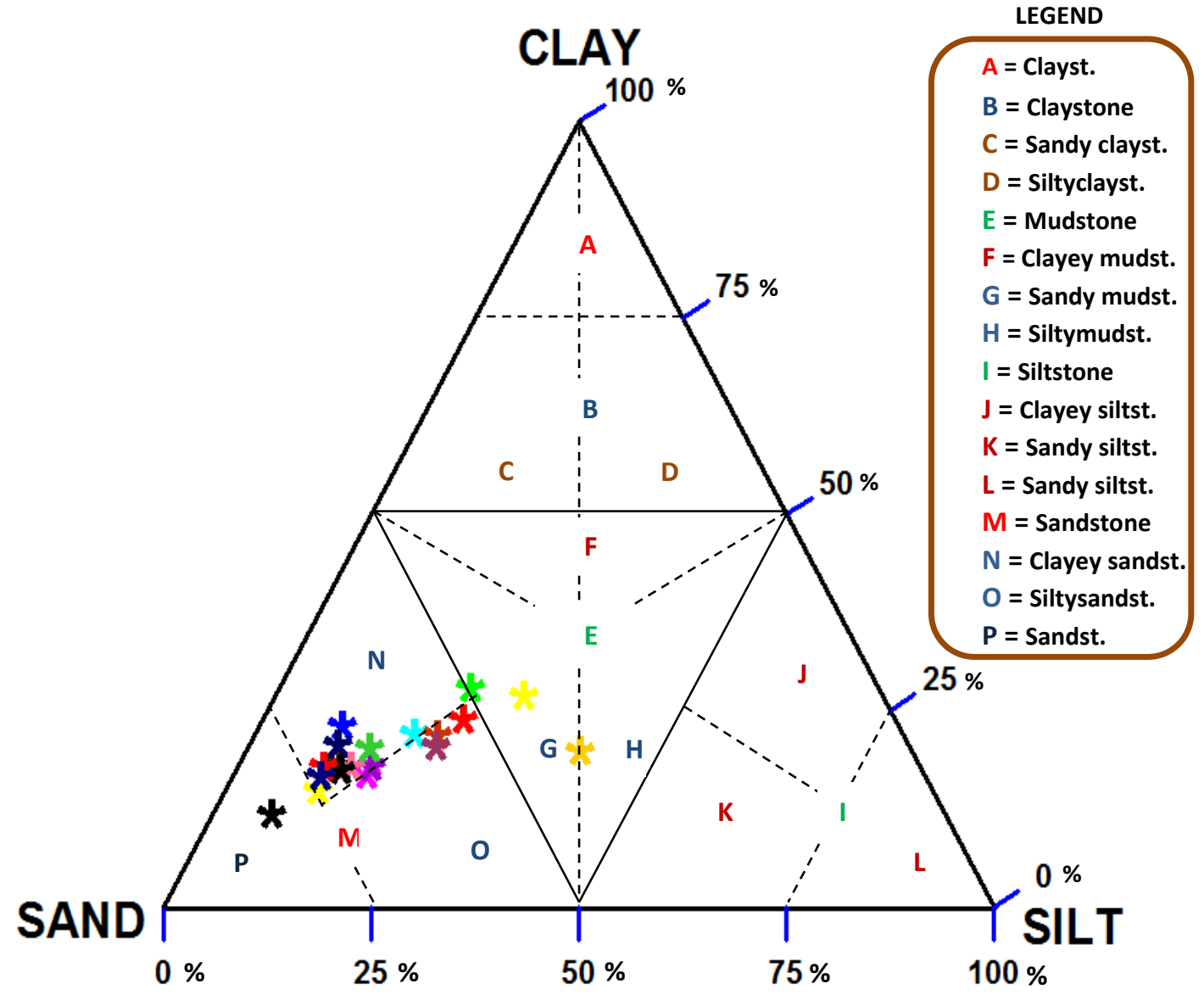

Fig. 2: Ternary plot showing Lithologic distribution of sediments 
Table 2: Lithologic Description of the Epe Lagoon Sediments

\begin{tabular}{|c|c|c|c|c|c|c|}
\hline $\mathbf{S} / \mathbf{N}$ & $\begin{array}{l}\text { SAMPLE } \\
\text { NUMBER } \\
\end{array}$ & FORMATION & LITHOLOGY & TEXTURE & COLOUR & $\begin{array}{l}\text { ORGANIC } \\
\text { MATTER }\end{array}$ \\
\hline 1 & Epe/st/1b & Coastal-plain sands & Clayey-sand & Fine grain & Dark Grey & $\begin{array}{l}\text { Presence of fossil } \\
\text { shells }\end{array}$ \\
\hline 2 & Epe/st/10a & Coastal-plain sands & Clayey-sand & Fine & Grey & $\begin{array}{l}\text { No visible organic } \\
\text { material found }\end{array}$ \\
\hline 3 & Epe/st/7a & Coastal-plain sands & Clayey-sand & Fine-medium & Grey & $\begin{array}{l}\text { Presence of fossil } \\
\text { shells }\end{array}$ \\
\hline 4 & Epe/st/4b & Coastal-plain sands & Clayey-sand & Fine & Light grey & $\begin{array}{l}\text { Presence of fossil } \\
\text { shells }\end{array}$ \\
\hline 5 & Epe/st/1a & Coastal-plain sands & Clayey-sand & Fine & Dark grey & $\begin{array}{l}\text { Presence of fossil } \\
\text { shells }\end{array}$ \\
\hline 6 & Epe/st/4a & Coastal-plain sands & Clayey-sand & Fine & Dark grey & $\begin{array}{l}\text { Presence of fossil } \\
\text { shells and organic } \\
\text { debris }\end{array}$ \\
\hline 7 & Epe/st/8b & Coastal-plain sands & Clayey-sand & Fine & Light & $\begin{array}{l}\text { Presence of fossil } \\
\text { shells }\end{array}$ \\
\hline 8 & Epe/st/3a & Coastal-plain sands & Clayey-sand & Fine-medium & Grey & $\begin{array}{l}\text { Presence of fossil } \\
\text { shells }\end{array}$ \\
\hline 9 & Epe/st/5b & Coastal-plain sands & Clayey-sand & Fine-medium & Grey & $\begin{array}{l}\text { presence of fossil } \\
\text { shells }\end{array}$ \\
\hline 10 & Epe/st/7b & Coastal-plain sands & Clayey-sand & Fine-medium & Grey & $\begin{array}{l}\text { Presence of fossil } \\
\text { shells }\end{array}$ \\
\hline 11 & Epe/st/3b & Coastal-plain sands & Clayey-sand & Fine-medium & Grey & $\begin{array}{l}\text { Presence of fossil } \\
\text { shells }\end{array}$ \\
\hline 12 & Epe/st/9a & Coastal-plain sands & Clayey-sand & Fine-medium & Dark grey & $\begin{array}{l}\text { Presence of fossil } \\
\text { shells and organic } \\
\text { debris }\end{array}$ \\
\hline 13 & Epe/st/9b & Coastal-plain sands & Clayey-sand & Fine & Dark grey & $\begin{array}{c}\text { Presence of fossil } \\
\text { shells and organic } \\
\text { debris }\end{array}$ \\
\hline 14 & Epe/st/5a & Coastal-plain sands & Clayey-sand & Fine-medium & Grey & $\begin{array}{c}\text { Presence of fossil } \\
\text { shells }\end{array}$ \\
\hline 15 & Epe/st/2a & Coastal-plain sands & Clayey-sand & Fine & Light grey & $\begin{array}{l}\text { No visible organic } \\
\text { material found }\end{array}$ \\
\hline 16 & Epe/st/8a & Coastal-plain sands & Clayey-sand & Fine & Grey & $\begin{array}{l}\text { Presence of fossil } \\
\text { shells }\end{array}$ \\
\hline 17 & Epe/st/6a & Coastal-plain sands & Clayey-sand & Fine & Light grey & $\begin{array}{c}\text { Presence of organic } \\
\text { debris }\end{array}$ \\
\hline 18 & Epe/st/6b & Coastal-plain sands & Clayey-sand & Fine & Grey & $\begin{array}{c}\text { Presence of organic } \\
\text { debris }\end{array}$ \\
\hline 19 & Epe/st/10b & Coastal-plain sands & Clayey-sand & Fine & Light grey & $\begin{array}{c}\text { Presence of organic } \\
\text { debris }\end{array}$ \\
\hline
\end{tabular}

of $66 \%$ by weight of the upper continental crust (Taylor and McLennan, 1985). Corresponding high average value of $\mathrm{Al}_{2} \mathrm{O}_{3} /\left(\mathrm{Na}_{2} \mathrm{O}+\mathrm{CaO}\right)$ recorded as 138.099 (Table 5) which is a ratio of the most immobile element to the most mobile element confirms the high content of clastic materials over carbonate materials within the sediment. Also, $\mathrm{Al}_{2} \mathrm{O}_{3} /\left(\mathrm{Fe}_{2} \mathrm{O}_{3}+\mathrm{MgO}\right)$ values which ranged from (1.65-29.54) (Table 5) indicates relatively moderate to high digenetic alteration. Furthermore, $\mathrm{K}_{2} \mathrm{O} / \mathrm{Al}_{2} \mathrm{O}_{3}$ values were found to range from 0.002-0.4973 suggesting sedimentary recycling or increase in the degree of source area weathering (Bauluz et.al, 2000); it also indicates weathering and alteration (Table 6). Very low average values of $\mathrm{Al}_{2} \mathrm{O}_{3} / \mathrm{SiO}_{2}$ recorded as 0.08932 is an indication of quartz enrichment (Bhatia, 1983), this supports the relatively high average value of $\mathrm{SiO}_{2} / \mathrm{Al}_{2} \mathrm{O}_{3}$ recorded as 64.33 (Table 5) which also confirms the high $\mathrm{SiO}_{2}$ values previously observed within the sediments. Values of $\mathrm{Al}_{2} \mathrm{O}_{3}, \mathrm{Fe}_{2} \mathrm{O}_{3}$ and $\mathrm{MgO}$ were observed to decrease with increasing $\mathrm{SiO}_{2}$, while others such as $\mathrm{CaO}, \mathrm{Na}_{2} \mathrm{O}, \mathrm{K}_{2} \mathrm{O}, \mathrm{TiO}_{2}$, $\mathrm{P}_{2} \mathrm{O}_{5}$ and $\mathrm{MnO}$ had very low concentrations (Table 3). More so, $\mathrm{TiO}_{2} / \mathrm{Al}_{2} \mathrm{O}_{3}$ values ranged from $0.004-0.8604$, indicating that the sediments are chemically matured (Jenner et.al, 1981). The correlation results of $\mathrm{TiO}_{2}$ against $\mathrm{Al}_{2} \mathrm{O}_{3}$ and $\mathrm{Fe}_{2} \mathrm{O}_{3}$ with $\mathrm{Al}_{2} \mathrm{O}_{3}$ 
were positive $(\mathrm{r}=0.98$ and 0.60$)$, while $\mathrm{Na}_{2} \mathrm{O}$ with $\mathrm{Al}_{2} \mathrm{O}_{3}$ was negative $(\mathrm{r}=0.24)$ suggesting partial association with phyllosilicates (Fig. 3). The correlation results with $\mathrm{K}_{2} \mathrm{O}$ were also positive ( $r=0.22,0.14$ and 0.74$)$ suggesting the input of aluminosilicates and feldspar within the sediments. The positive correlation between $\mathrm{K}_{2} \mathrm{O}$ and $\mathrm{Al}_{2} \mathrm{O}_{3}(\mathrm{r}=0.19)$ (Fig. 3) implies that the concentration of potassium bearing minerals have significant influence on the distribution of aluminum (Al) and thus suggest that the abundance of these elements is controlled by the content of clay minerals (McLennan et. al, 1983).

Table 3: Geochemical Composition of Epe lagoon sediments (Major elements expressed in percentage)

\begin{tabular}{|c|c|c|c|c|c|c|c|c|c|c|c|c|c|}
\hline $\mathbf{S} / \mathbf{N}$ & SAMPLE & LOCATION & $\mathrm{SiO}_{2}$ & $\mathbf{A l}_{2} \mathbf{O}_{3}$ & $\mathrm{Fe}_{2} \mathrm{O}_{3}$ & MgO & $\mathrm{CaO}$ & $\mathrm{Na}_{2} \mathrm{O}$ & $\mathrm{K}_{2} \mathrm{O}$ & $\mathrm{TiO}_{2}$ & $\mathbf{P}_{2} \mathbf{O}_{5}$ & MnO & Total \\
\hline 1 & Epe/st/1b & Epe lagoon & 60.9 & 30.3 & 1.43 & 0.07 & 0.04 & 0.02 & 0.33 & 3.42 & 0.08 & 0.01 & 96.6 \\
\hline 2 & Epe/st/10a & Epe lagoon & 81.73 & 11.52 & 0.35 & 0.04 & 0.03 & 0.03 & 0.16 & 1.25 & 0.05 & 0.01 & 95.17 \\
\hline 3 & Epe/st/7a & Epe lagoon & 52.99 & 35.46 & 1.55 & 0.05 & 0.02 & 0.01 & 0.17 & 4.56 & 0.1 & 0.01 & 94.92 \\
\hline 4 & Epe/st/4b & Epe lagoon & 97.31 & 1.07 & 0.25 & 0.02 & 0.03 & 0.04 & 0.04 & 0.26 & 0.02 & 0.01 & 99.0 \\
\hline 5 & Epe/st/1a & Epe lagoon & 98.37 & 0.71 & 0.04 & 0.01 & 0.03 & 0.04 & 0.04 & 0.06 & 0.01 & 0.01 & 99.32 \\
\hline 6 & Epe/st/4a & Epe lagoon & 92.86 & 3.56 & 0.8 & 0.02 & 0.01 & 0.02 & 0.03 & 0.32 & 0.01 & 0.01 & 97.64 \\
\hline 7 & Epe/st/8b & Epe lagoon & 80.19 & 10.17 & 2.96 & 0.03 & 0.02 & 0.01 & 0.03 & 0.9 & 0.06 & 0.01 & 94.38 \\
\hline 8 & Epe/st/3a & Epe lagoon & 96.87 & 0.86 & 0.51 & 0.01 & 0.01 & 0.01 & 0.06 & 0.05 & 0.01 & 0.01 & 98.40 \\
\hline 9 & Epe/st/5b & Epe lagoon & 98.87 & 0.71 & 0.04 & 0.01 & 0.01 & 0.03 & 0.04 & 0.05 & 0.01 & 0.01 & 99.78 \\
\hline 10 & Epe/st/7b & Epe lagoon & 94.35 & 2.56 & 0.05 & 0.05 & 0.06 & 0.01 & 0.05 & 0.84 & 0.02 & 0.01 & 98.0 \\
\hline 11 & Epe/st/3b & Epe lagoon & 97.46 & 0.86 & 0.34 & 0.04 & 0.05 & 0.01 & 0.04 & 0.74 & 0.01 & 0.01 & 99.56 \\
\hline 12 & Epe/st/9a & Epe lagoon & 96.65 & 1.7 & 0.29 & 0.01 & 0.02 & 0.03 & 0.08 & 0.12 & 0.01 & 0.01 & 98.92 \\
\hline 13 & Epe/st/9b & Epe lagoon & 97.62 & 0.87 & 0.11 & 0.01 & 0.01 & 0.02 & 0.04 & 0.12 & 0.01 & 0.01 & 98.82 \\
\hline 14 & Epe/st/5a & Epe lagoon & 97.77 & 0.87 & 0.1 & 0.01 & 0.02 & 0.01 & 0.04 & 0.12 & 0.01 & 0.01 & 98.96 \\
\hline 15 & Epe/st/2a & Epe lagoon & 97.30 & 1.05 & 0.22 & 0.02 & 0.03 & 0.03 & 0.04 & 0.25 & 0.01 & 0.01 & 98.96 \\
\hline 16 & Epe/st/8a & Epe lagoon & 96.40 & 1.75 & 0.27 & 0.04 & 0.30 & 0.03 & 0.09 & 0.43 & 0.06 & 0.45 & 99.82 \\
\hline 17 & Epe/st/6a & Epe lagoon & 93.36 & 1.83 & 0.67 & 0.04 & 0.42 & 0.08 & 0.91 & 0.58 & 0.21 & 0.08 & 98.18 \\
\hline 18 & Epe/st/6b & Epe lagoon & 94.97 & 2.41 & 0.40 & 0.11 & 0.07 & 0.02 & 0.05 & 0.01 & 0.22 & 0.04 & 98.30 \\
\hline 19 & Epe/st/10b & Epe lagoon & 92.70 & 4.20 & 0.59 & 0.05 & 0.07 & 0.05 & 0.30 & 0.35 & 0.33 & 0.07 & 98.71 \\
\hline
\end{tabular}

Table 4: Summary of major oxide composition of the Epe-lagoon sediments

$\begin{array}{llllll}\text { Oxides } & \text { Range }(\%) & \text { Mean }(\mathbf{N}=19) & \begin{array}{l}\text { Standard } \\ \text { Error }\end{array} & \begin{array}{l}\text { Standard } \\ \text { Deviation }\end{array} & \text { Variance } \\ \mathrm{SiO}_{2} & 52.99-98.87 & 90.46 & 2.97 & 12.93 & 167.20 \\ \mathrm{Al}_{2} \mathrm{O}_{3} & 0.71-35.46 & 5.92 & 2.30 & 10.01 & 100.26 \\ \mathrm{Fe}_{2} \mathrm{O}_{3} & 0.04-2.96 & 0.58 & 0.16 & 0.72 & 0.52 \\ \mathrm{MgO} & 0.01-0.11 & 0.03 & 0.006 & 0.03 & 0.001 \\ \mathrm{CaO} & 0.01-0.42 & 0.099 & 0.04 & 0.18 & 0.033 \\ \mathrm{Na} 2 & 0.01-0.08 & 0.026 & 0.004 & 0.02 & 0.000 \\ \mathrm{~K}_{2} \mathrm{O} & 0.03-0.91 & 0.13 & 0.048 & 0.20 & 0.043 \\ \mathrm{TiO}_{2} & 0.01-4.56 & 0.76 & 0.28 & 1.20 & 1.45 \\ \mathrm{P}_{2} \mathrm{O}_{5} & 0.01-0.33 & 0.07 & 0.021 & 0.09 & 0.008 \\ \mathrm{MnO} & 0.01-0.45 & 0.04 & 0.23 & 0.10 & 0.010\end{array}$


Table 5: Major element ratios for sediments of the Epe lagoon, Eastern Dahomey basin, Nigeria

\begin{tabular}{|c|c|c|c|c|c|c|c|}
\hline S/NO & SAMPLE & $\mathrm{SiO}_{2} / \mathrm{Al}_{2} \mathrm{O}_{3}$ & $\mathrm{Fe}_{2} \mathrm{O}_{3} / \mathrm{K}_{2} \mathrm{O}$ & $\mathrm{Na}_{2} \mathrm{O}+\mathrm{CaO}$ & $\mathrm{Al}_{2} \mathrm{O}_{3} /\left(\mathrm{Na}_{2} \mathrm{O}+\mathrm{CaO}\right)$ & $\mathrm{Fe}_{2} \mathrm{O}_{3}+\mathrm{MgO}$ & $\mathrm{Al}_{2} \mathrm{O}_{3} /\left(\mathrm{Fe}_{2} \mathrm{O}_{3}+\mathrm{MgO}\right)$ \\
\hline 1 & Epe/st/1b & 2.01 & 4.33 & 0.06 & 505 & 1.5 & 20.2 \\
\hline 2 & Epe/st/10a & 7.10 & 2.19 & 0.06 & 192 & 0.39 & 29.54 \\
\hline 3 & Epe/st/7a & 1.49 & 9.12 & 0.03 & 1182 & 1.6 & 22.16 \\
\hline 4 & $\mathrm{Epe} / \mathrm{st} / 4 \mathrm{~b}$ & 95.40 & 6.25 & 0.07 & 14.57 & 0.27 & 3.78 \\
\hline 5 & Epe/st/1a & 138.55 & 1 & 0.07 & 10.14 & 0.05 & 14.2 \\
\hline 6 & $\mathrm{Epe} / \mathrm{st} / 4 \mathrm{a}$ & 26.08 & 26.67 & 0.03 & 118.67 & 0.82 & 4.34 \\
\hline 7 & $\mathrm{Epe} / \mathrm{st} / 8 \mathrm{~b}$ & 7.89 & 98.67 & 0.03 & 339 & 2.99 & 3.40 \\
\hline 8 & Epe/st/3a & 112.64 & 8.5 & 0.02 & 43 & 0.52 & 1.65 \\
\hline 9 & Epe/st/5b & 139.25 & 1 & 0.04 & 17.75 & 0.05 & 14.2 \\
\hline 10 & $\mathrm{Epe} / \mathrm{st} / 7 \mathrm{~b}$ & 36.86 & 1 & 0.07 & 36.57 & 0.1 & 25.6 \\
\hline 11 & $\mathrm{Epe} / \mathrm{st} / 3 \mathrm{~b}$ & 113.33 & 8.5 & 0.06 & 14.33 & 0.38 & 2.26 \\
\hline 12 & Epe/st/9a & 56.86 & 3.63 & 0.05 & 34 & 0.3 & 5.67 \\
\hline 13 & Epe/st/9b & 112.21 & 2.75 & 0.03 & 29 & 0.12 & 7.25 \\
\hline 14 & Epe/st/5a & 112.38 & 2.5 & 0.03 & 29 & 0.11 & 7.91 \\
\hline 15 & $\mathrm{Epe} / \mathrm{st} / 2 \mathrm{a}$ & 92.67 & 5.5 & 0.06 & 17.5 & 0.24 & 4.38 \\
\hline 16 & Epe/st/8a & 55.09 & 3 & 0.33 & 5.30 & 0.31 & 5.65 \\
\hline 17 & Epe/st/6a & 51.02 & 0.74 & 0.5 & 3.66 & 0.71 & 2.58 \\
\hline 18 & Epe/st/6b & 39.41 & 8 & 0.09 & 26.78 & 0.51 & 4.73 \\
\hline 19 & Epe/st/10b & 22.07 & 1.97 & 0.75 & 5.6 & 0.64 & 6.56 \\
\hline
\end{tabular}

Table 6: Major element ratios for Epe lagoon sediments

\begin{tabular}{|c|c|c|c|c|c|c|}
\hline S/NO & SAMPLE & $\mathrm{K}_{2} \mathrm{O} / \mathrm{Al}_{2} \mathrm{O}_{3}$ & $\mathrm{TiO}_{2} / \mathrm{Al}_{2} \mathrm{O}_{3}$ & $\mathrm{~K}_{2} \mathrm{O}+\mathrm{CaO}+\mathrm{MgO}$ & $\mathrm{Fe}_{2} \mathrm{O}_{3}+\mathrm{MgO}$ & $\overline{\mathrm{Al}_{2} \mathrm{O}_{3} / \mathrm{SiO}_{2}}$ \\
\hline 1 & Epe/st/1b & 0.011 & 0.11 & 0.44 & 1.5 & 0.498 \\
\hline 2 & Epe/st/10a & 0.014 & 0.11 & 0.23 & 0.39 & 0.141 \\
\hline 3 & Epe/st/7a & 0.005 & 0.13 & 0.24 & 1.6 & 0.669 \\
\hline 4 & Epe/st/4b & 0.039 & 0.25 & 0.09 & 0.27 & 0.010 \\
\hline 5 & Epe/st/1a & 0.056 & 0.08 & 0.08 & 0.05 & 0.007 \\
\hline 6 & Epe/st/4a & 0.008 & 0.09 & 0.06 & 0.82 & 0.038 \\
\hline 7 & Epe/st/8b & 0.003 & 0.09 & 0.08 & 2.99 & 0.127 \\
\hline 8 & Epe/st/3a & 0.070 & 0.06 & 0.08 & 0.52 & 0.009 \\
\hline 9 & Epe/st/5b & 0.056 & 0.07 & 0.06 & 0.05 & 0.007 \\
\hline 10 & Epe/st/7b & 0.020 & 0.33 & 0.16 & 0.1 & 0.027 \\
\hline 11 & Epe/st/3b & 0.047 & 0.86 & 0.13 & 0.38 & 0.009 \\
\hline 12 & Epe/st/9a & 0.047 & 0.07 & 0.11 & 0.3 & 0.018 \\
\hline 13 & Epe/st/9b & 0.046 & 0.14 & 0.06 & 0.12 & 0.009 \\
\hline 14 & Epe/st/5a & 0.046 & 0.14 & 0.07 & 0.11 & 0.009 \\
\hline 15 & Epe/st/2a & 0.038 & 0.24 & 0.09 & 0.24 & 0.011 \\
\hline 16 & Epe/st/8a & 0.051 & 0.25 & 0.43 & 0.31 & 0.018 \\
\hline 17 & Epe/st/6a & 0.050 & 0.32 & 1.37 & 0.71 & 0.020 \\
\hline 18 & Epe/st/6b & 0.021 & 0.004 & 0.23 & 0.51 & 0.025 \\
\hline 19 & Epe/st/10b & 0.071 & 0.08 & 1.05 & 0.64 & 0.045 \\
\hline
\end{tabular}



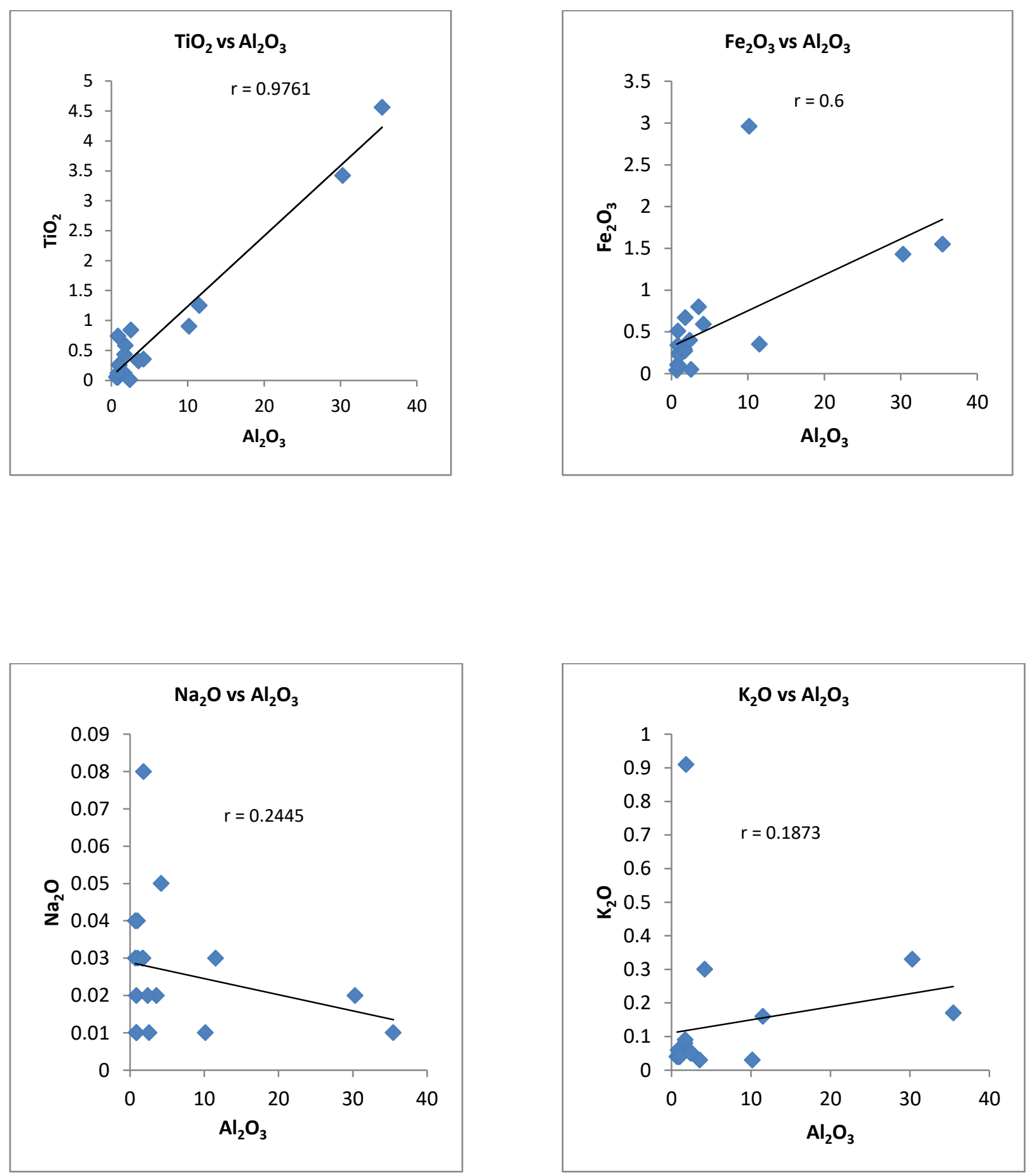

Fig. 3: Correlation plot of major oxides 


\section{Classification of the Epe lagoon sediments}

Standard plot of Herron (1988) using $\log \left(\mathrm{Fe}_{2} \mathrm{O}_{3} / \mathrm{K}_{2} \mathrm{O}\right)$ against $\log \left(\mathrm{SiO}_{2} / \mathrm{Al}_{2} \mathrm{O}_{3}\right)$ which is the modified version of Pettijohn et.al, (1972) was employed to classify the sediments of the Epe lagoon. However, it was observed that about one-third of the sediments plotted within the quartz arenite region which is an indication of quartz enrichment and a confirmation of the high $\mathrm{SiO}_{2}$ values recorded from the major element geochemistry results (Table 3). However, a notable fraction of the sediments also plotted within the Sublitharenite and Subarkose region indicating a change in the level of sediment maturity. Minor fractions of the Epe lagoon sediments also plotted randomly within the Fe-shale, Fe-sand, shale and wacke regions (Fig. 4).

\section{Paleo-Weathering Indices and Maturity}

The Chemical Index of Alteration (CIA) and the Plagioclase Index of Alteration (PIA) are the most widely used indices for quantitative estimation of the degree of chemical weathering undergone by rocks of the provenance area of clastic sediments (Fedo et.al, 1995). CIA records the alkali, and alkali earth elements (Nesbitt and Young, 1982); this is because alteration of igneous rocks during weathering results in depletion of alkali and alkaline earth elements and preferential enrichment of $\mathrm{Al}_{2} \mathrm{O}_{3}$ in sediments. Some other indices which may also be used include Chemical Indices of Weathering (CIW) (Harnois, 1988). Summary of CIA, PIA and PIW values are shown in Table 7. $\mathrm{CIA}=100\left\{\mathrm{Al}_{2} \mathrm{O}_{3} /\left(\mathrm{Al}_{2} \mathrm{O}_{3}+\mathrm{CaO}+\mathrm{Na}_{2} \mathrm{O}+\mathrm{K}_{2} \mathrm{O}\right)\right\}$

$\mathrm{PIA}=100\left\{\left(\mathrm{Al}_{2} \mathrm{O}_{3}-\mathrm{K}_{2} \mathrm{O}\right) /\left(\mathrm{Al}_{2} \mathrm{O}_{3}+\mathrm{CaO}+\mathrm{Na}_{2} \mathrm{O}-\mathrm{K}_{2} \mathrm{O}\right)\right\}$

$\mathrm{CIW}=100\left\{\mathrm{Al}_{2} \mathrm{O}_{3} /\left(\mathrm{Al}_{2} \mathrm{O}_{3}+\mathrm{CaO}+\mathrm{Na}_{2} \mathrm{O}\right)\right\}$

Nesbitt and young (1982) suggested that high values (76-100) indicate intensive chemical weathering at the source areas while low values (50 or less) indicate unweathered source areas. Furthermore, Osae et.al, (2006) also suggested that high CIA and PIA values (75-100) indicate intensive weathering in the source area whereas low values $(<60)$ indicates low weathering in source area. Generally, the sediments of the Epe lagoon has CIA, PIA and CIW values ranging from (56.48-99.44, 64.79-99.92, and 78.54-99.92) with an average of (90.44, 93.53, and 94.47) respectively (Table 7). This results indicates high weathering at source areas as suggested by Nesbitt and young (1982) and Osae et.al, (2006). Also the CIA values were plotted in the (A-CN-K) diagram (Fig. 5), in the diagram the sediments plotted mostly within the kaolinite-chlorite and illite region. The fact that the samples did not plot very close to the plagioclase and K-feldspar line confirms also that the Epe lagoon sediments have undergone high (intensive) weathering at the source area.

\section{Depositional Environment of the Epe lagoon sediments}

The depositional environment of the Epe lagoon sediments was obtained using the ternary plots of Englung and Jorgensen (1973) which was done on the basis of the $\left(\mathrm{Al}_{2} \mathrm{O}_{3}\right)-\left(\mathrm{K}_{2} \mathrm{O}+\mathrm{Na}_{2} \mathrm{O}+\mathrm{CaO}\right)-$ $\left(\mathrm{Fe}_{2} \mathrm{O}_{3}+\mathrm{MgO}\right)$ contents. As observed from the plots, most of the sediments deposited within the continental zone and are argillaceous (Fig. 6). However, there is a fairly gradual transition of some of the sediments from the continental environments into the transitional environment. More so, some of the sediments within the continental zone also plotted within the region of ferruginous argillites. 


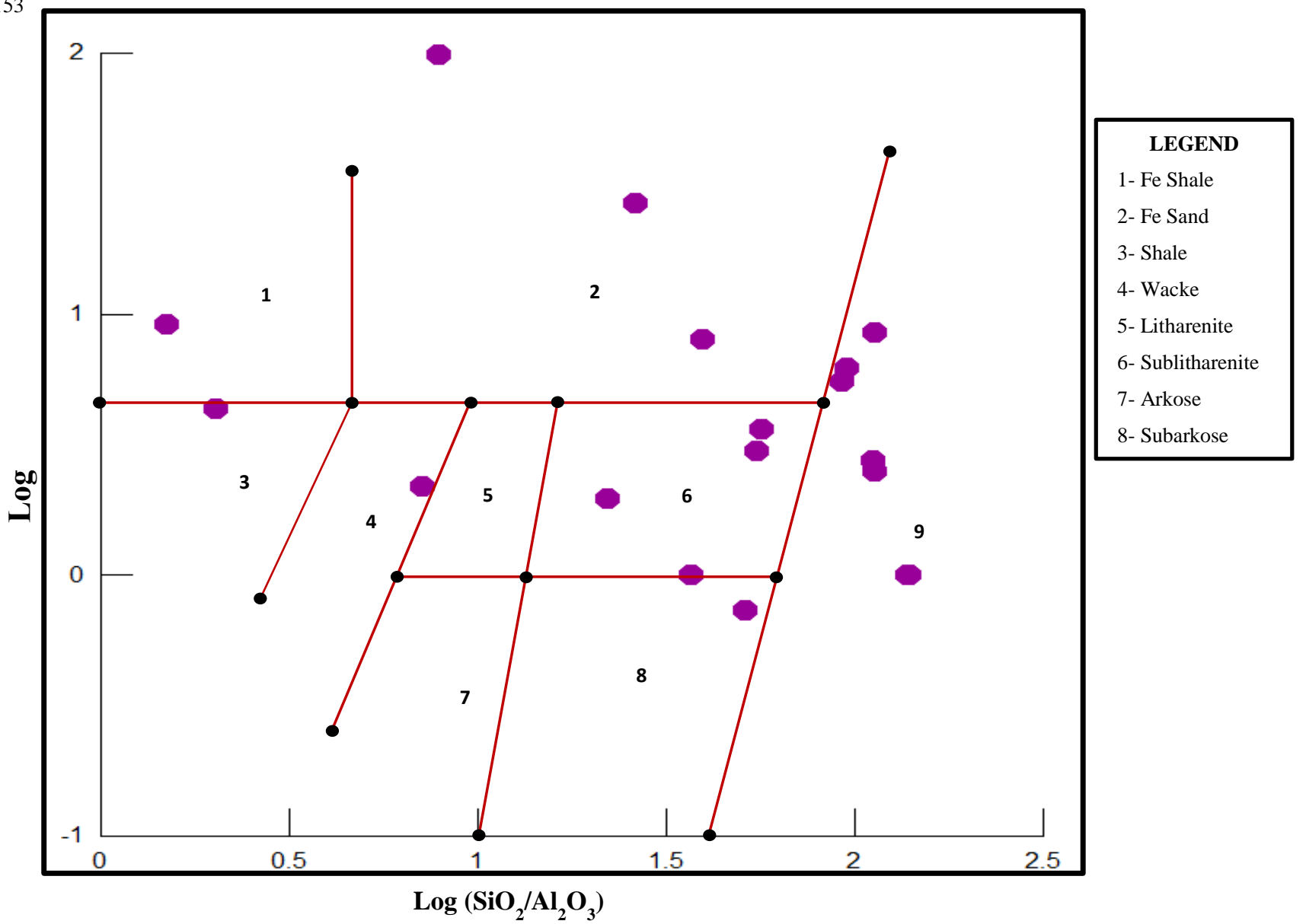

Fig. 4: Classification of Epe lagoon sediments (modified after Herron, 1988)

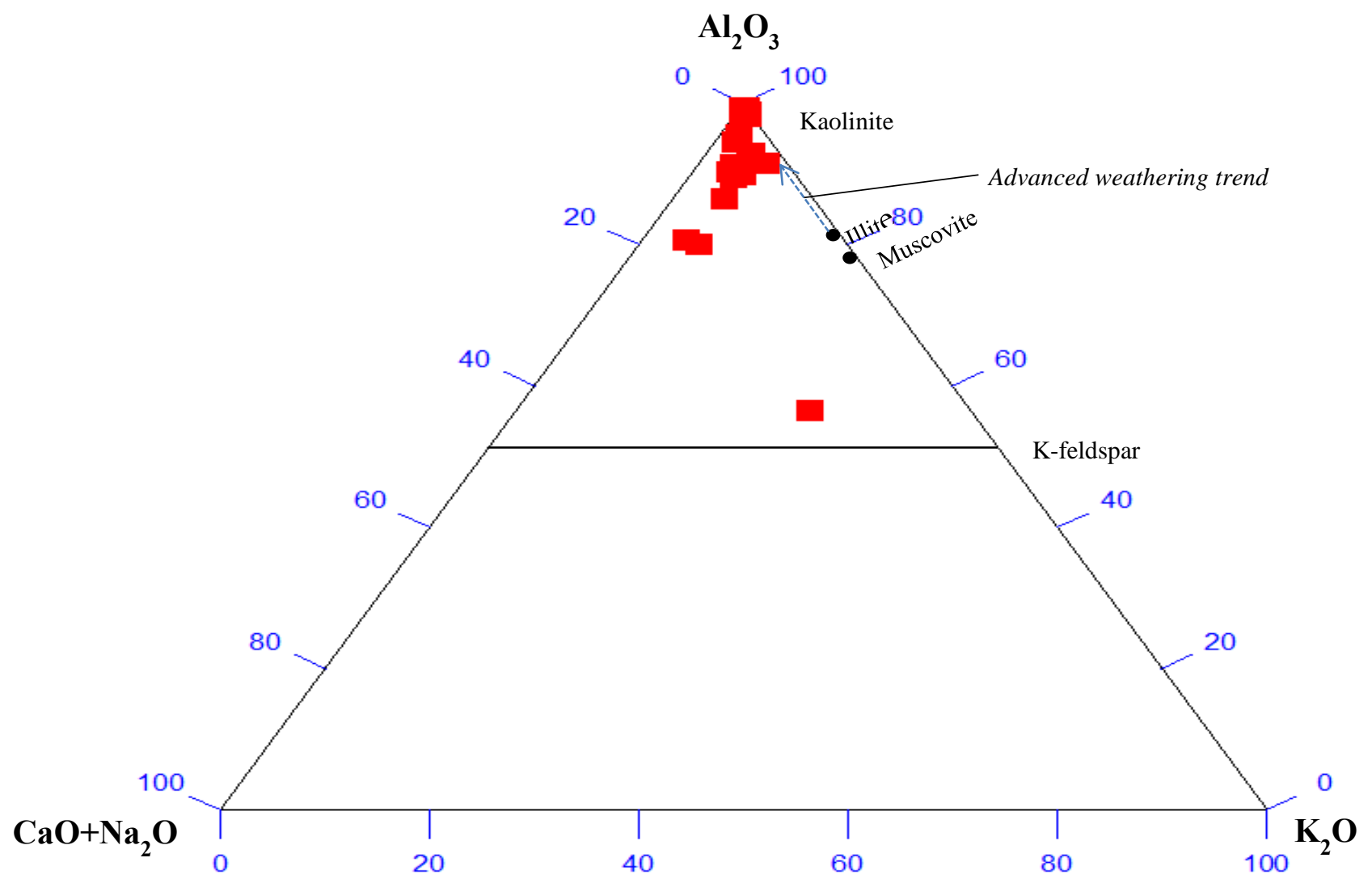

Fig. 5: CIA ternary diagram $\left(\mathrm{Al}_{2} \mathrm{O}_{3}\right)-\left(\mathrm{CaO}+\mathrm{Na}_{2} \mathrm{O}\right)-\left(\mathrm{K}_{2} \mathrm{O}\right)$ for the Epe lagoon sediments (after Nesbitt and young, 1982) 
Table 7: CIA, PIA and CIW values for sediments of the Epe lagoon

\begin{tabular}{|c|c|c|c|c|}
\hline S/N & SAMPLE NUMBER & CIA & PIA & CIW \\
\hline 1 & Epe/st/1b & 98.73 & 99.80 & 99.80 \\
\hline 2 & Epe/st/10a & 98.13 & 99.47 & 99.48 \\
\hline 3 & Epe/st/7a & 99.44 & 99.92 & 99.92 \\
\hline 4 & Epe/st/4b & 90.27 & 93.33 & 93.58 \\
\hline 5 & Epe/st/1a & 86.59 & 90.54 & 91.03 \\
\hline 6 & Epe/st/4a & 98.34 & 99.16 & 99.16 \\
\hline 7 & Epe/st/8b & 99.41 & 99.71 & 99.71 \\
\hline 8 & Epe/st/3a & 91.49 & 97.56 & 97.73 \\
\hline 9 & Epe/st/5b & 89.87 & 94.37 & 94.67 \\
\hline 10 & Epe/st/7b & 95.52 & 97.29 & 97.34 \\
\hline 11 & Epe/st/3b & 89.58 & 93.18 & 93.48 \\
\hline 12 & Epe/st/9a & 92.90 & 97.01 & 97.14 \\
\hline 13 & Epe/st/9b & 92.55 & 96.51 & 96.67 \\
\hline 14 & Epe/st/5a & 92.55 & 96.51 & 96.67 \\
\hline 15 & Epe/st/2a & 91.30 & 94.39 & 94.59 \\
\hline 16 & Epe/st/8a & 80.65 & 83.42 & 84.13 \\
\hline 17 & Epe/st/6a & 56.48 & 96.79 & 78.54 \\
\hline 18 & Epe/st/6b & 94.51 & 83.87 & 96.40 \\
\hline 19 & Epe/st/10b & 80.0 & & 84.85 \\
\hline
\end{tabular}

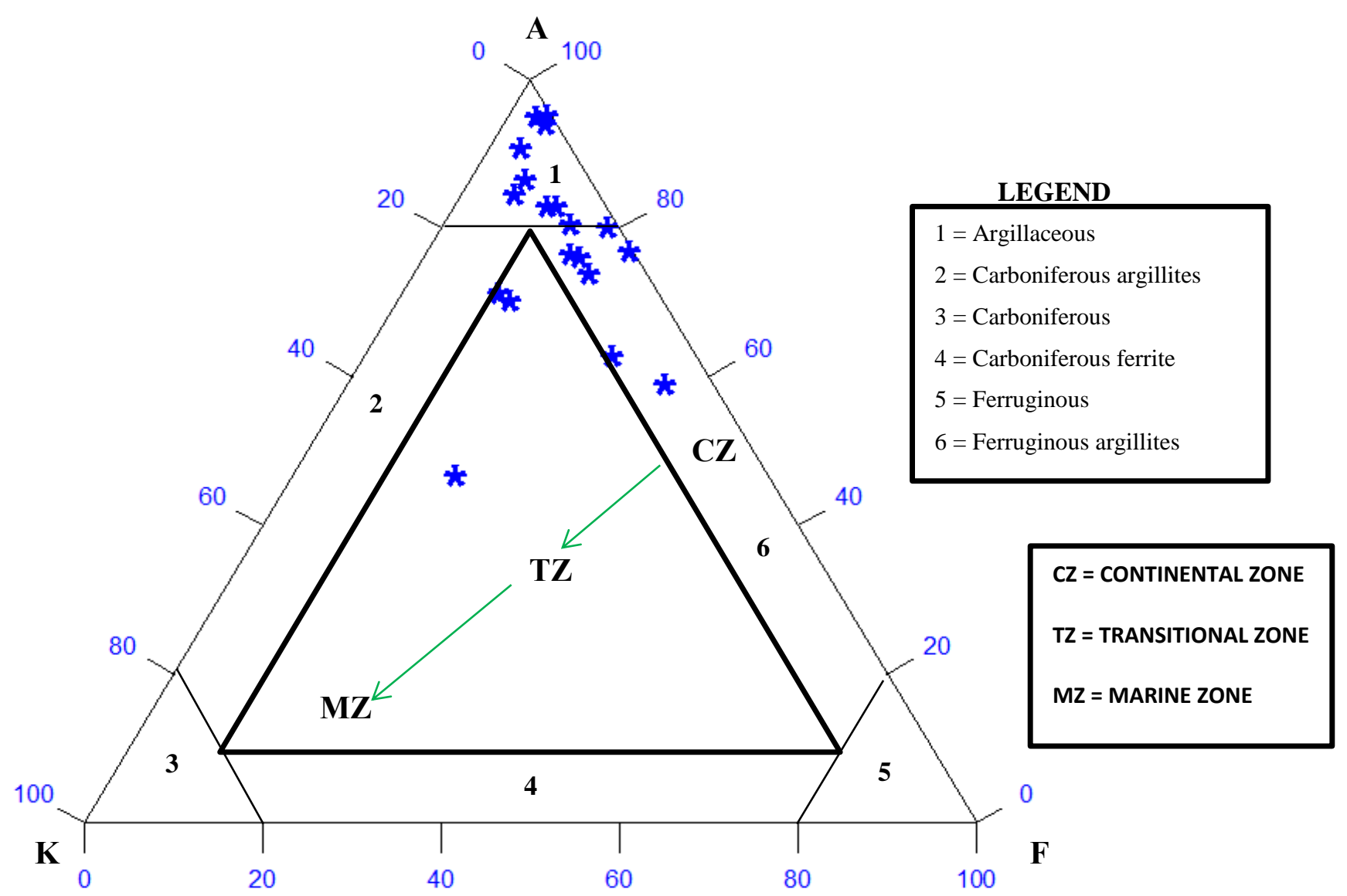

Fig. 6: $\mathrm{Al}_{2} \mathrm{O}_{3}-\left(\mathrm{K}_{2} \mathrm{O}+\mathrm{CaO}+\mathrm{MgO}\right)-\left(\mathrm{Fe}_{2} \mathrm{O}_{3}+\mathrm{MgO}\right)\{\mathrm{AKF}\}$ Ternary plot for Epe lagoon sediments (after Englung and Jorgensen, 1973) 


\section{CONCLUSION}

The inorganic geochemical analysis of the Epe lagoon revealed that $\mathrm{SiO}_{2}$ is the dominant oxide, followed by $\mathrm{Al}_{2} \mathrm{O}_{3}$ and $\mathrm{Fe}_{2} \mathrm{O}_{3}$ which constitutes over $90 \%$ of the total oxides. Others are $\mathrm{MgO}$, $\mathrm{CaO}, \mathrm{Na}_{2} \mathrm{O}, \mathrm{K}_{2} \mathrm{O}, \mathrm{TiO}_{2}$ and $\mathrm{P}_{2} \mathrm{O}_{5}$. The $\mathrm{SiO}_{2} / \mathrm{Al}_{2} \mathrm{O}_{3}$ ratios for the sediments were appreciably high indicating that the sediments have been heavily weathered and transported; this is also corroborated by the enrichment of quartz and depletion feldspar in most of the samples. The fairly high $\mathrm{K}_{2} \mathrm{O} / \mathrm{Na}_{2} \mathrm{O}$ ratios may be attributed to the presence of plagioclase within the sediments. From the major element geochemistry, the application of source area weathering using CIA, CIW and PIA with mean values of 90.44, 93.53 and 94.47 respectively indicates that the sediments of the Epe lagoon have been subjected to intense weathering at the source area as well as high levels of chemical weathering and transportation. In the A-CN-K diagram the sediments of the Epe lagoon plotted mostly within the kaolinite-chlorite and illite region; also an indication of high weathering at the source.

The use of heron's model classified the sediments as mainly quartz arenite. However, a notable fraction of the sediments were also as Sublitharenite and Subarkose with very minor fractions classified as Fe-shale, Fe-sand and Wacke. AKF ternary plots revealed that the sediments were deposited mainly in continental environment and dominated by argillaceous materials with some ferruginous argillites, although some fractions of the sediments were also found to have been transported from the continental environment to the transition zone.

\section{APPENDIX}

\section{INORGANIC GEOCHEMISTRY}

Determination of Major element abundances using the Atomic Absorption Spectrophotometry (AAS) Method

Atomic absorption spectrophotometry is based upon the observation that atoms of an element can absorb electromagnetic radiation. This occurs when the element is atomized and the wavelength of light absorbed is specific to each element. Thus, the atomic absorption spectrophotometer comprises an atomizing device, during the atomization of a sample in a beam of light, as a consequence of atomic absorption, can be calibrated and is sensitive at the ppm level. Each sample was first pulverized and $0.5 \mathrm{gram}$ digested with a mixture of prepared solution of Nitric acid and Hydrochloric acid (ratio 1:3); also known as Aqua regia solution. The sample was stirred and heated inside a fume cupboard. The digested sample was diluted with distilled water and made up to $20 \mathrm{ml}$ mark. About $1 \mathrm{ml}$ was taken from the solution and further diluted with distilled water to $10 \mathrm{ml}$ mark, which represents the stock solution, $\mathrm{x}$ 10-dilution factor. The major elements $\mathrm{Si}, \mathrm{Al}, \mathrm{Fe}, \mathrm{Mn}, \mathrm{Mg}, \mathrm{Ca}, \mathrm{Na}, \mathrm{K}$ and $\mathrm{Ti}$ were then determined from the digested samples using a Perkin Elmer 400 atomic absorption spectrophotometer. These elements were further converted from ppm level to their respective oxides in percentage. The AAS is a widely used technique and comparatively cheap. However, there are two limitations to the routine use of AAS in silicate analysis. Firstly, the sample must be prepared as a solution, and secondly it is element-specific, i.e. one element can usually be analyzed at a time, although this latter limitation has in part been overcome by fitting instruments with multiple-turret lamp holders.

\section{REFERENCES}

Adegoke, O.S. 1969. Eocene Stratigraphy of Southern Nigeria. Mem. Bur. Geol. Minieres. 69:23-49.

Adediran, S.A. and Adegoke, O.S. 1987. Evolution of the Sedimentary Basins of the Gulf of Guinea. In: Current Research in Africa Earth Sciences, Balkema, Rotterdam. Matheis and Schandeimeier. Eds. 283-286.

Aderinola, O.J., Clarke, E.O., Olarinmoye, M.O., Kusemiju, V. and Anatekhai, M.A. 2009. Heavy metals in surface water, sediments, fish and periwinkles of Lagos lagoon. American-Eurasian Journal of Agriculture and Environmental Science. Vol 5(5): 609-617.

Agagu, O.k. 1985. A geological guide to Bituminous sediments of South-western Nigeria. Published by the Geology Department. University of Ibadan, Ibadan. 24pp.

Avramidis, P., Panagiotaras, D., Papoulis, D., and Koutopoulos, N. 2010. Sedimentological and Geochemical characterization of holocence sediments from Alikes lagoon, Zakynthos Island, Western Greece. Bulletin of the Geological Society of Greece. Proceedings of the $12^{\text {th }}$ international congress.

Ayoola, S.O. and Kuton, M.P. 2009. Seasonal variation in Fish abundance and physicochemical parameters of Lagos lagoon, Nigeria. African Journal of Environmental Science and Technology. Vol 3(5):149-156.

Badejo, O.T., Olaleye, J.B., and Alademomi, A.S. 2014. Tidal characteristics and sounding datum variation in Lagos state. International Journal of Innovative Research and studies.

Balogun, K.J., Ladigbolu, I.A., and Ariyo, A.A. 2011. Ecological assessment of a coastal shallow lagoon in Lagos, Nigeria: A bio-indicator approach. J.Appl.sci. Environ.manage. Vol 15(1) 41-46.

Bauluz, B., Mayaye, M.J., Fernando-Nieto, C. and Lopez, M.G. 2000. Geochemistry of Precambrian and Paleozoic siliclastic rocks from Iberia (N/E Spain); Implications for source area weathering, sorting, provenance and tectonic setting. Chemical Geology. 135-150.

Billman, H.G. 1992. Offshore stratigraphy and paleontology of the Dahomey embayment, West Africa. Nigerian Association of Petroleum Explorationist (NAPE) Bulletin. Vol 7(2): 121-130. 
Don-Pedro, K.N., Oyewo, E.O. and Otitoloju, A.A. 2004. Trend of heavy metal concentrations in Lagos lagoon ecosystem, Nigeria. West African Journal of Applied Ecology. 5:103-114

Englund, J.O. and Jorgensen, P. 1973. A chemical classification system for Argillaceous sediments and factors affecting their composition. Geol. Stockholm Forh. 95:87-97.

McLeanan, S.M., Taylor., S.R. and Eriksson, K.A. 1983. Geochemistry of archean shales from the Pilbara super group, western Australia. Geochim. Cosmochim. Acta. 47:1211-1222.

Mpanda, S. 1997. Geological development of East African coastal Basin of Tanzania; Acta Universities Stockholmiensis. Vol 45. 121pp.

Nesbitt, H.W. and Young, G.M. 1982. Early Proterozoic climate and plate motion inferred from major element chemistry of Latites. Journal of Nature. 299:715-717

Ojeda, H.A. 1982. Structural framework, stratigraphy and evolution of Brazilian Basins. American Association of Petroleum Geologist Memoir. 66:732 749

Olatunji, A.S., and Abimbola, A.F. 2010. Geochemical Evaluation of the Lagos Lagoon sediments and water. World Applied Science Journal 9(2): 178193.

Omatsola, M.E. and Adegoke, O.S. 1981. Tectonic evolution and cretaceous stratigraphy of the Dahomey Basin. Journal of Mining and Geology. Vol 18(1):130-137.

Osae, S., Asiedu, D.K., Banoeng-Yakubo, B., Koekerl, C. and Dampare, S.B. 2006. Provenance and tectonic setting of late Proterozoic Buem sandstones of South-eastern Ghana; evidence from geochemistry and detrital modes. Journal of African Earth Science. 44:85-96.

Slansky, M. 1962. Contribution a pelude geologique du Basin sedimentaire cotier au Dahomey at du Togo. Mem Bur.Rech. Geolo. Min. 11.

Storey, B.C. 1995. The role of mantle plumes in continental break up; case history from Gondwanaland. Journal of Nature. 377:301-308.

\section{AUTHORS}

First Author - OMOREGBE Osagie Aret, OND, HND (Petroleum Training Institute), B.Sc., (University of Ibadan), M.Sc., (University of Benin) Pg Dip (Laser Petroleum Geoscience Centre).Omoregbeosagie88@gmail.com

Second Author - OLOYEDE, David Abiodun, B.Sc., (University of Ilorin), M.Sc., (University of Benin)
Harnois, L. 1988. The new index, a new Chemical index of weathering. Sedimentary geology. Vol 55: 319-322.

Jenner, G.A., Firyer, B.J. and Mclennan, S.M. 1981. Geochemistry of Archean yellow knife super group. Geochin. Cosmochin. Acta. Vol 45:1111-1129.

Ogbe, F.A.G., 1972. Stratigraphy of strata exposed in the Ewekoro quarry, western Nigeria. In: T.F.G Dessauvagie and A.J Whiteman. Eds. African Geology, Ibadan University Press. 305-322.

Ogunsua, A.O., Adebona, M.B., Ako-Nai, K.A., Oluwole, A.F., Ruck, W. and Oyewo, E.O. 1993. Metal in the Lagos lagoon. In; proceedings of the international conference on heavy metals in the environment, Toronto, Canada. R.J. Allan and J.O. Nriagu. 165-168.

Okoye, B.C.O., Afolabi, O.A. and Ajao, E.O. 1991. Heavy metals in the Lagos lagoon sediments. International Journal of Environmental Studies. $37: 35-41$

Osibanjo, O. and Bamgbose, O. 1990. Chlorinated hydrocarbons in Marine fish and Shellfish of Nigeria. Mar. Pollut. Bull. 27:581-586.

Oyewo, E.O., Ajao, E.A. and Orekoya, T. 1982. Seasonal variations in surface temperature and salinity around Lagos harbor, Nigeria. National Institute of Marine and Oceanography Research (NIOMR) technical paper. No.10: $20 \mathrm{pp}$.

Third Author - EKUERUGBE, Lucky Orezimena, B.Sc., (Federal University of Petroleum Resources), M.Sc., (University of Benin)

Fourth Author - NKIM, Akere Godson, B.Sc., (University of Port Harcourt), M.Sc., (University of Port Harcourt)

.Correspondence Author - OMOREGBE Osagie Aret, Omoregbeosagie88@gmail.com, 08138820324. 